\title{
Strong atmospheric disturbances as a possible origin of inner zone particle diffusion
}

\author{
O. A. Pokhotelov ${ }^{1}$, V. A. Pilipenko ${ }^{1}$, M. Parrot $^{2}$ \\ ${ }^{1}$ Institute of the Physics of the Earth, Moscow 123810, Russia \\ ${ }^{2}$ Laboratoire de Physique et Chimie de l'Environnement, CNRS, 3A av. de la Recherche Scientifique, F-45071 Orléans, France, e-mail: \\ mparrot@cnrs-orleans.fr
}

Received: 4 May 1998 / Revised: 1 October 1998 / Accepted: 13 October 1998

\begin{abstract}
A new mechanism of the atmosphere-magnetosphere interaction, which might be called "acousticmagnetospheric cyclotron accelerator", is proposed. The idea of this mechanism stems from the fact that strong acoustical perturbations in the ionosphere (e.g., due to earthquakes, thunderstorms, etc.) may generate magnetic disturbances in the magnetosphere. Then, the latter will induce local resonant acceleration and subsequent inward diffusion of trapped particles. This idea may be fruitful in the interpretation of some occasional increases in inner zone particle fluxes which do not correlate with the solar or magnetospheric activities.
\end{abstract}

Key words. Ionosphere (active experiments; ionosphereatmosphere interactions; particle acceleration)

\section{Introduction}

Experimental evidence is mounting for the mutual influence of processes in different geophysical media. Consequently a modern understanding of geophysical phenomena requires consideration of the effects in more than one medium. Some effects of the mutual influence in the lithosphere-atmosphere-ionosphere-magnetosphere system are known and have been extensively studied:

1. Ionosphere-magnetosphere coupling - ionospheremagnetosphere interaction is an essential part of aurora dynamics in the night-side magnetosphere (Goertz and Boswell, 1979).

2. Technogenic influence on the ionosphere - modification of the ionosphere caused by additional precipitation of magnetospheric energetic particles induced by radio emission from ground radio transmitters,

Correspondence to: M. Parrot industrial facilities, etc. (Fraser-Smith, 1981; Parrot and Zaslavski, 1996).

3. Meteorological control of the ionosphere - dependence of the parameters of the lower ionosphere on large-scale motions of neutral atmosphere (Danilov et al., 1987).

4. Terrogenic effects in the ionosphere - influence of the Earth's crustal features on the dynamical processes in the ionosphere (Popov et al., 1989).

5. Ionospheric precursors of earthquakes - influence of seismic-related electromagnetic phenomena on the VLF noise background and on the ionospheric parameters (Gokhberg et al., 1995; Gal'perin et al., 1992; Molchanov et al., 1995; Hayakawa et al., 1996).

It is impossible to disregard the effects of the interaction between different geophysical domains and, though some progress in this direction has been made, all these effects should be studied further. The present paper addresses one more possible effect of geophysical media interaction: additional radial diffusion and acceleration of inner zone particles, induced by strong acoustical motions in the upper atmosphere. The proposed mechanism of particle acceleration and inward diffusion is related to transformation of acoustical wave energy into MHD modes in the ionospheric E-layer. Essentially, according to our estimates, the ground effect of excited MHD disturbances is small, whereas in the magnetosphere the corresponding magnetic disturbance may have substantial magnitude. Finally, we discuss some experimental indications on the feasibility of the considered mechanism.

\section{Physical mechanism of MHD wave generation in the ionosphere by an acoustic disturbance}

The key element of the scenario in hand lies in the fact that atmosphere-magnetosphere interaction results in the excitation of electric currents and magnetic fields by acoustic disturbances in the ionospheric $E$-layer. The problem of magnetic disturbance generation by acous- 
tical waves has been already treated within the framework of different theoretical models (Belov et al., 1973; Al'perovich et al., 1979; Jacobson and Bernhardt, 1985; Borisov and Moiseyev, 1989; Pokhotelov et al., 1994). At a more simple and physically obvious level, it is attempted to demonstrate in the following the general idea of acoustic-magnetohydrodynamic coupling in the mid-latitude ionosphere.

Various natural and man-made phenomena in the lower atmosphere can be a source of strong acoustical disturbances, e.g. severe tropospheric weather storms, Rayleigh surface waves or tsunami from earthquakes, volcano eruptions, large rocket launches, flights of supersonic aircrafts or chemical and nuclear explosions (for references see reviews by Karlov et al., 1980; Blanc, 1985; Al'perovich et al., 1985; Pokhotelov et al., 1995; Guglielmi and Pokhotelov, 1996). Emitted acoustic waves increase in amplitude as they propagate upward through the atmosphere, passing progressively through layers with exponentially decreasing density. In this process the upper atmosphere acts as a filter, allowing only a certain frequency band of acoustic noise to pass. At low-frequency edge atmospheric pass-band filter is limited by the cut-off frequency. The high-frequency cutoff is controlled by a stronger attenuation of higher frequencies $\left(\sim f^{2}\right)$ due to dissipation processes. Doppler soundings of the $E$ - and $F$-layers (Prasad et al., 1975; Okuzawa et al., 1983, 1986) actually showed that the ionospheric response constituted band-limited oscillations with prevailing periods from several seconds up to several minutes.

In the ionospheric plasma, where collisions do occur, the motion of neutral particles transverse to the geomagnetic field line forces the ionized particles to oscillate; hence currents are induced. These oscillating currents act as a source for MHD waves, which then may propagate along the ionosphere or into the magnetosphere.

At first, it seems that the transformation must be very weak due to the enormous difference between the Alfvén velocity in the ionosphere, $c_{A} \simeq 5 \cdot 10^{2} \mathrm{~km} / \mathrm{s}$, and the sound velocity $c_{s} \simeq 5 \cdot 10^{-1} \mathrm{~km} / \mathrm{s}$. Actually, the pressure variation $\delta p$ in acoustic wave yields to the relationship:

$\delta p / p=v_{n} / c_{s}$,

where $v_{n}$ is the perturbation of a neutral particle bulk velocity and $p$ stands for the unperturbed pressure. The disturbed magnetic field $\delta B$ in the MHD wave can be estimated as follows

$\delta B / B \simeq v_{i} / c_{A}$,

where $v_{i}$ is the velocity of the ionized plasma component and $B$ stands for the unperturbed magnetic field (for these rough estimates we neglect the difference between $v_{E}, v_{i}$, and $v_{e}$ ).

For the most favorable conditions, i.e. when $v_{n} \sim v_{i}$, the transformation coefficient $T$ becomes equal to

$T \equiv(\delta B / B) /(\delta p / p) \simeq c_{S} / c_{A} \sim 10^{-3}$.
Thus, when an acoustic impulse with an amplitude of $\delta p / p \sim 10^{-1}$ enters the ionosphere, it would generate magnetic disturbances with an amplitude no larger than several $n \mathrm{~T}$; that is $\delta B \sim 3 n \mathrm{~T}$ for $B=3 \times 10^{4} n \mathrm{~T}$ (Danilov and Dovzhenko, 1987). The above elementary consideration illustrates the basic problem of an efficient coupling between the acoustic waves and MHD disturbances.

However, in the ionospheric plasma the properties of MHD waves are modified considerably, as compared to collisionless plasma. The simplest way to understand the properties of these waves is to consider their propagation along a magnetic field $\mathbf{B}$ in a homogeneous plasma, where all the disturbances can be considered as plane waves

$(\delta \mathbf{B}, \mathbf{E}) \propto \exp (-i \omega t+i \mathbf{k} \cdot \mathbf{r})$,

propagating along the field lines, where $\omega$ is the wave angular frequency, and $\mathbf{k}$ is the wave vector.

In the frequency range $v_{i n} \ll \omega \ll \omega_{c i}$ (where $v_{i n}$ is the ion-neutral collision frequency and $\omega_{c i}$ is the ion angular gyrofrequency), which holds in the ionized plasma of the upper ionosphere, a wave propagates with the ordinary Alfvén velocity $c_{A}$. The neutrals with density $N_{n}$ are practically not disturbed by the wave. As the frequency decreases, the MHD wave becomes attenuated due to ion-neutral collisions.

At very low frequencies, when $v_{n i} \sim \omega \ll \omega_{c i}\left(v_{n i}\right.$ is a neutral-ion collision frequency: $v_{n i}=\left(N_{0} / N_{n}\right) v_{i n}$, where $N_{0}$ is a total ion density), multicomponent plasma starts to move as a whole, without slipping between the velocities of ionized and neutral plasma components. It was suggested to call this wave regime a "slow MHD wave" (Sorokin and Fedorovich, 1982), which is not to be confused with the slow ion acoustic mode. The phase velocity of the wave for the case when ions drag the neutrals, i.e. when $v_{n} \sim v_{i}$, turns out to be (Al'perovich et al., 1979)

$v_{p h}=B /\left(4 \pi M_{i} N_{n}\right)^{1 / 2}=c_{A}\left(N_{0} / N_{n}\right)^{1 / 2}$.

However, in the realistic ionosphere, even at altitudes of the $F$-layer, the expected periods of the slow MHD disturbances are too long, that is about several hours.

In the $E$-layer, where the plasma conductivity transverse to the magnetic field becomes highly anisotropic (local Hall conductivity is much larger than Pedersen conductivity), a new modified MHD mode appears, called the gyrotropic MHD mode (Sorokin and Fedorovich, 1982). The phase velocity of this gyrotropic MHD mode in the non-dissipative plasma differs essentially from the ordinary Alfvén velocity:

$v_{p h}=c_{A}\left(\omega / \omega_{c i}\right)^{1 / 2}$

in the range of parameters $\omega_{c e} \gg v_{e n}$ ( $v_{e n}$ is the electron-neutral collision frequency and $\omega_{c e}$ is the electron gyrofrequency) and $\omega \ll \omega_{c i} \ll v_{i n}$, that are typical for the $E$-layer. A characteristic feature of the gyrotropic MHD mode, namely the velocity decrease by a factor $\left(\omega / \omega_{c i}\right)^{1 / 2}$, predicts a high transformation coefficient, up to $T \sim 5 \times 10^{-2}$, in the $E$-layer in accordance with Eq. (3). For this case magnetic distur- 
bances with amplitudes up to hundreds of $n \mathrm{~T}$ may be expected; e.g. for $\delta p / p \sim 10^{-1}$ and $B=3 \times 10^{4} n \mathrm{~T}$, $\delta B \sim 1.5 \times 10^{2} n \mathrm{~T}$. The above simplified consideration should be considered only as some intuitive guess, indicating that in the ionospheric $E$-layer the coupling between an acoustic impact and MHD disturbances might be substantial. In fact, more advanced theoretical models (Belov et al., 1973; Pokhotelov et al., 1994) support this notion.

But the most convincing argument, in our opinion, is presented by the experimental results obtained during the MASSA experiment (Gal'perin et al., 1985; Gal'perin and Hayakawa, 1996). The low-orbiting Aureol-3 satellite actually recorded magnetic impulses in the upper ionosphere with amplitudes of $10^{2} n \mathrm{~T}$ resulting from ground-based explosions. Polarization of the electromagnetic impulse showed that it was an Alfvén wave, propagating upwards from the ionosphere. Then, Alfvén impulse oscillated between the conjugate ionospheres and shifted due to dispersion to lower $L$-shells. As a result, the lower latitude magnetosphere became a "well" for Alfvén disturbances that were triggered by the ionospheric acoustical motion.

\section{Magnetospheric and ground effects of magnetic disturbances induced by an acoustic wave}

After all the above considerations a very appropriate question arises: if the magnetic disturbances induced by acoustic waves might be so strong, why only small effects are observed by ground-based magnetometers, particularly after explosions and earthquakes (Al'perovich et al., 1985)? To answer this question we now estimate the effects expected above the ionosphere and on the ground.

Since we are only interested in the region near the source, we may limit our analysis to a quasi-static approximation. We suppose that in the northern ionosphere a neutral velocity disturbance $v_{n}$ arises, perpendicular to $\mathbf{B}$, with a wave vector $k_{x}=k$ (Fig. 1). We also assume, that the disturbance scale in the vertical direction $z$ is larger than the scale of the current-carrying layer of the ionosphere.

Then from the electrodynamic point of view, the ionosphere can be imagined as a thin film with an anisotropic tensor conductivity:

$\hat{\Sigma}=\left(\begin{array}{cc}\Sigma_{P} & \Sigma_{H} \\ -\Sigma_{H} & \Sigma_{P}\end{array}\right)$

where the subscripts $P$ and $H$ refer to Pedersen and Hall height-integrated conductivities, respectively. The electric field $\mathbf{E}^{(N)}$ in the northern ionosphere is comprised of the dynamo and polarization fields according to:

$\mathbf{E}_{\perp}^{(N)}=-\nabla_{\perp} \varphi^{(N)}+\mathbf{v}_{n} \times \mathbf{B}$.

The local ionosphere is connected by equipotential field lines to the conjugate southern ionosphere, i.e. $\varphi^{(N)}=\varphi^{(S)}$, while $\mathbf{E}_{\perp}^{(S)}=-\nabla_{\perp} \varphi^{(S)}$. Now, proceeding from the current conservation condition:

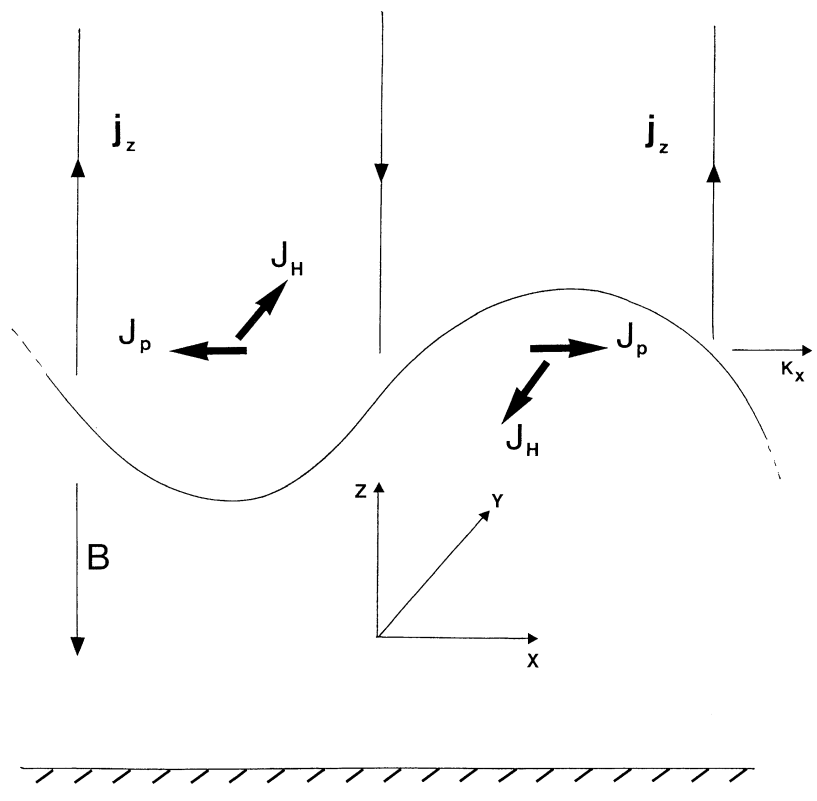

Fig. 1. Simplified picture of the current system, excited by a periodic acoustic motion, and constituted with field-aligned $\left(j_{z}\right)$, Pedersen $\left(J_{P}\right)$ and Hall $\left(J_{H}\right)$ currents

$j_{z}{ }^{(N, S)}=-\nabla \cdot \mathbf{J}_{\perp}{ }^{(N, S)}$,

and Ohm's law for the height-integrated transverse current $\mathbf{J}_{\perp}$

$\mathbf{J}_{\perp}{ }^{(N, S)}=\hat{\Sigma} \cdot \mathbf{E}_{\perp}{ }^{(N, S)}$,

we may obtain the equations for the potential $\varphi$ of the polarization field

$\nabla \cdot\left(\hat{\Sigma} \cdot \nabla_{\perp} \varphi\right)=\frac{1}{2} \nabla \cdot\left[\hat{\Sigma} \cdot\left(\mathbf{v}_{n} \times \mathbf{B}\right)\right]$,

and for the field-aligned current

$j_{z}=\frac{B}{2}\left[\Sigma_{P}\left(\nabla \times \mathbf{v}_{n}\right)_{z}-\Sigma_{H} \nabla \cdot \mathbf{v}_{n}\right]$.

From Eq. (12) it follows that the field-aligned current $j_{z}$ can be excited either by an acoustical wave (for which $\left(\nabla \times \mathbf{v}_{n}\right)_{z}=0$ and $\left.\nabla \cdot v_{n} \neq 0\right)$ due to Hall conductivity, or by a non-compressional gravity wave (for which $\left(\nabla \times \mathbf{v}_{n}\right)_{z} \neq 0$ and $\left.\nabla \cdot v_{n}=0\right)$ due to Pedersen conductivity.

The polarization of horizontally stratified $F$-region plasma and perturbations of plasma density induced by acoustic and gravity waves were also studied in electrostatic approximation by Jacobson (1986), and Jacobson and Bernhardt (1985), who demonstrated that at low latitudes electrostatic effects should be mirrored in the conjugated ionosphere.

We further concentrate on estimates of the current, induced by an acoustical wave, in the $E$-layer. For the qualitative estimates let us limit ourselves by considering the simple one-dimensional model $(\mathrm{d} / \mathrm{dy}=0)$ illustrated in Fig. 1.

The transverse current can be decomposed into the potential (Pedersen) and the solenoidal (Hall) parts with the potentials $\Phi$ and $\Psi$, respectively: 
$J_{\perp}=-\nabla_{\perp} \Phi+(\hat{\mathbf{z}} \times \nabla) \Psi$,

where $\hat{\mathbf{z}}$ is the unit vector along the ambient magnetic field, i.e. $\hat{\mathbf{z}} \equiv \mathbf{B} / B$.

According to Fukushima's (1969) theorem the resultant effect of field-aligned and Pedersen currents is zero at the ground. The magnetic disturbance at the Earth's surface is only produced by the Hall part containing the $\Psi$ potential.

The magnetic disturbance in the magnetosphere can be calculated from the Ampére's law and Eq. (12), and results in

$\delta B_{y} / B=-\left(\mu_{0} / 2\right) \Sigma_{H} v_{n}$

where $\mu_{0}$ is the permeability of a free space.

Then, for the $\Psi$ part we obtain from Eqs. (10)-(13)

$$
\begin{aligned}
\nabla_{\perp} \Psi=\left(\nabla \times J_{\perp}\right)_{z}=-\frac{B}{2}\{ & \Sigma_{H}\left(\nabla \times \mathbf{v}_{n}\right)_{z} \\
& \left.+\left(2 \Sigma_{P}+\frac{\Sigma_{H}^{2}}{\Sigma_{P}}\right) \nabla \cdot \mathbf{v}_{n}\right\}
\end{aligned}
$$

Finally, for the Hall current induced by the acoustic wave we have:

$J_{y}=-B v_{n}\left(\Sigma_{P}+\frac{\Sigma_{H}^{2}}{2 \Sigma_{P}}\right)$.

The ground magnetic effect from the current system in hand is given by:

$\delta B_{x}=\frac{\mu_{0} J_{y}}{\pi h^{2}} \zeta K_{1}(\zeta)$

where $K_{1}(\zeta)$ is the modified Bessel function, $\zeta=k h$, and $h$ is the height of the current carrying layer.

Estimates, which follow from Eqs. (16) and (17) for $\Sigma_{H}=2 \Sigma_{P}, \quad \Sigma_{P}=10 \quad \mathrm{~S}, \quad B=3 \times 10^{4} n \mathrm{~T}, \quad h=100 \mathrm{~km}$, $v_{n}=50 \mathrm{~m} / \mathrm{s}$ and $k=2 \pi / 100 \mathrm{~km}^{-1}$ yield $\delta B_{y} \simeq 0.5 \times 10^{2} n \mathrm{~T}$ in the magnetosphere and $\delta B_{x} \simeq 3 n \mathrm{~T}$ on the ground. The given estimates show that the ground magnetic effect of the ionospheric currents induced by an acoustic impulse is small as compared with that in the magnetosphere. The reason of this fact is that a satellite detects the direct effect of a localized field-aligned current, while ground-based magnetometer records the spatially integrated effect of small-scale ionospheric current system.

\section{Inner zone particle acceleration and diffusion}

Now we consider what kind of influence may be exerted on the energetic trapped particles from locally excited hydromagnetic disturbances. This process has to be described by a diffusion-type system of equations in the quasi-linear approximation. Just for simplicity, our consideration of the effect is based on the relationships for typical wave and particle parameters, which actually are the characteristics of the relevant quazi-linear differential equations.

Due to the conservation of the first two adiabatic invariants the radial displacement $\delta L$ of trapped parti- cles is directly related to the variation of energy $\delta W$ as follows (Lyons and Williams, 1984):

$\delta W / W=-3 \delta L / L$.

For simplicity herein we only consider the particles mirroring near the geomagnetic equator. As it can be deduced from Eq. (18) any transfer of energy from MHD disturbances to particles will be accompanied by their inward radial diffusion. The energy imparted to the charged non-relativistic particles in a time-varying field can be estimated from the well known formula (e.g. Sturrock, 1994):

$\delta W=\int \mathrm{d} t\left(e v_{d} E_{\phi}+\mu \frac{\partial \delta B_{\|}}{\partial t}\right)$.

Here $\delta B_{\|}$is field aligned perturbation of the magnetic field, $e$ is the magnitude of the particle charge, $\mu$ is the particle magnetic moment, $v_{d}$ is the azimuthal drift velocity, $E_{\phi}$ is the azimuthal component of the wave electric field, and integration is made along particle trajectories. The appearance of the second term in the brackets on the right side of Eq. (19) is connected with the fact, that in the time-dependent magnetic field, there must be an accompanying electric field according to the Faraday's law.

If we expand the wave fields $\mathbf{E}$ and $\delta \mathbf{B}$ into Fourier integral over time $t$ and azimuthal angle $\phi$, then for particles moving along trajectories $\phi(t)=\phi_{0}+\omega_{d} t$, where $\phi_{0}$ is the initial particle phase and $\omega_{d}$ is the magnetic drift frequency, the energy gain per one pass through the disturbed region will be

$\delta W=\int_{-\infty}^{\infty} \mathrm{d} m \delta W_{m}$

where

$$
\begin{aligned}
\delta W_{m}=\int \mathrm{d} t & {\left[e v_{d} E_{\phi}(m, \omega)-i \mu \omega \delta B_{\|}(m, \omega)\right] } \\
& \times \exp \left[-i\left(\omega-m \omega_{d}\right) t+i m \phi_{0}\right]
\end{aligned}
$$

where $m$ is the azimuthal wave number, and the superscripts in the initial values are omitted. Remembering that

$$
\int_{-\infty}^{\infty} \mathrm{d} t^{\prime} \exp \left(-i \alpha t^{\prime}\right)=\delta(\alpha),
$$

we obtain

$$
\begin{aligned}
\delta W=\int \mathrm{d} m\left[e v_{d} E_{\phi}(m, \omega)-i \mu \omega \delta B_{\|}(m, \omega)\right] \\
\times \delta\left(\omega-m \omega_{d}\right) \exp \left(i m \phi_{0}\right) .
\end{aligned}
$$

The appearance of a delta function in Eq. (23) means that the average energy gain is non-vanishing only for resonant particles. The resonance condition $\omega=m \omega_{d}$ means that an energy transfer from the wave to the particles occurs only when the period of the wave is 
equal to multiples of the particle drift period. The factor $\exp \left(i m \phi_{0}\right)$ relates to the proper phasing between the particles and the oscillating field: depending on the initial phase some particles will give energy to the wave, while others will extract energy from the wave. Further we will consider Eq. (23) only averaged over group of particles with such phases that $\delta W \geq 0$.

Now we consider the particle acceleration induced by a localized disturbance, such as

$$
\left(\mathbf{E}, \delta B_{\|}\right) \propto \exp \left[-(\phi / \Delta \phi)^{2}-i \omega t\right],
$$

where $\Delta \phi$ stands for the azimuthal extent of the source in hand.

For such disturbance we have from Eqs. (22) and (23)

$$
\begin{aligned}
\delta W= & \frac{\sqrt{\pi} \Delta \phi}{\omega_{d}}\left[e v_{d} E_{\phi}-i \omega \mu \delta B_{\|}\right] \\
& \times \exp \left[-\left(\frac{\omega \Delta \phi}{2 \omega_{d}}\right)^{2}+i \frac{\omega \phi_{0}}{\omega_{d}}\right] .
\end{aligned}
$$

The expression in Eq. (25) shows that the group of particles with energies such that the period of transit through a disturbed region is about the disturbance period will be resonantly accelerated in the most effective way.

A typical period of magnetic drift of non-relativistic particles in the inner magnetosphere can be estimated as (Lyons and Williams, 1984):

$\tau(\mathrm{mn}) \simeq 44 / L W(\mathrm{MeV})$.

The actual resonant period depends, as follows from Eq. (25), on the scale of disturbance localization. For example, an acoustically generated disturbance with the periods $\tau \sim 1 \mathrm{mn}$ and localized in the region $(\Delta \phi) L R_{E} \sim 10^{3} \mathrm{~km}$ can resonantly interact with electrons of $\mathrm{MeV}$ energy.

Scaling of Eq. (25) gives an estimate for resonant particle energy gain per one revolution

$$
\delta W\left(E_{\perp}\right) / W \simeq\left(c_{A} / \omega R\right)\left(\delta B_{\perp} / B\right), \quad \delta W\left(\delta B_{\|}\right) / W \simeq \delta B_{\|} / B
$$

Supposing that the curvature radius of geomagnetic field $R \simeq R_{E}, \quad \omega \simeq 10^{-1} s^{-1}, \quad c_{A} \simeq 3 \times 10^{3} \mathrm{~km} / \mathrm{s}, \quad$ and $B=$ $10^{4} n \mathrm{~T}$, for the disturbance with magnitude $\delta B_{\perp} \simeq 10 n \mathrm{~T}$ we get from Eq. (27) $\delta W / W \simeq 5 \times 10^{-3}$. An evaluation of the total energy gain $\delta W$ and consequent particle displacement $\delta L$ may require the consideration of several drift periods. For $N=5$ periods the relationship Eq. (27) would yield a value between $10^{-2}-10^{-1}$. So, the considered disturbance will accelerate some group of electrons up to additional energies $3 \times 10^{2} \mathrm{keV}$.

As was indicated by Falthammar (1965) particle acceleration in the magnetosphere via magnetic drift resonance is physically similar to the particle acceleration in a cyclotron or to the transit-time heating in a stellarator. Following Falthammar (1965) the discussed above process may be called "acoustic-magnetospheric cyclotron acceleration".

\section{Discussion}

The mechanism of electron acceleration presented above has much in common with that proposed by Cladis (1966) for the interpretation of the transient fluxes of $0.75 \mathrm{MeV}$ electrons observed by Imhof and Smith (1965) in the inner magnetosphere at $L=1.15$. In his approach, Cladis (1966) suggested the locally increased equatorial electrojet variations as an origin of $\mathrm{MeV}$ electron acceleration. In due course, we propose oscillations of the ionosphere-magnetosphere current system, induced by acoustical oscillatory motion, as an origin of the similar effects.

A particular source of acoustical disturbances may be, for example, surface Rayleigh waves emanating from earthquakes or severe weather storms. Preliminary analysis of data from the Nimbus-6 satellite indicated the existence of certain correlation of seismic activity with sporadic bursts of electrons in the gap between inner edge of inner radiation belt and atmosphere (Stassinopoulos and Vette, private communication, 1981). Similar effects were noticed by Gal'per et al. (1989) on board the MIR station, by Pustovetov and Malyshev (1993) on board the Meteor-3 satellite, and by Bosková et al. (1994) in the course of the processing the $I C-19$ data.

The comparison of electron precipitation from radiation belts as measured by Gamma satellite with the occurrence of tropical cyclones was made by Melioransky et al. (1996). They noticed that tropical cyclones are regularly accompanied by electron precipitation, whereas the maximum of precipitation corresponds to the most intense phase of cyclone evolution.

The result of resonant periodic action on the trapped radiation will be the formation of nearly monoenergetic group of electrons. Electron distribution function of this type ("bump on the tail" distribution) will be unstable under excitation of some high-frequency electrostatic turbulence. Thus, the described scenario represents the channel of transformation of the large-scale atmospheric motion into small-scale plasma turbulence in the magnetosphere. Then low-latitude regions of strong thunderstorm activity may be one of the sources of equatorial electrostatic VLF hiss, observed on board the $O G O-6$ satellite (Laaspere et al., 1971).

An interesting sub-class of near-equatorial electrostatic VLF hiss was discovered by IC-19 low-orbiting satellite (Larkina et al., 1983, 1988; Molchanov et al., 1993). The $L$-shell projection of this noise corresponded to the earthquake epicenters. Electrostatic noise lasted for many hours after a seismic shock. We may speculate that the process of energy transformation following the scheme "acoustical disturbance $\rightarrow$ MHD wave $\rightarrow$ electron acceleration $\rightarrow$ electrostatic VLF noise generation" may be responsible for the events observed on board $I C-19$ satellite.

\section{Conclusion}

The additional possible mechanism of the atmospheremagnetosphere coupling is proposed. Strong acoustic 
oscillations, reaching the ionospheric $E$-layer, may result in the generation of field-aligned currents. The magnetic effect of these currents in the magnetosphere might have a considerable magnitude $\left(\sim 10-10^{2} n \mathrm{~T}\right)$, whereas the ground signature of these magnetic disturbances is essentially weaker $(\sim$ several $n \mathrm{~T})$. Alfvén waves, generated by acoustic disturbances in the upper atmosphere, may induce resonant energizing and subsequent diffusion across $L$-shells of particles, trapped in the magnetosphere. As a whole, a physical picture described above represents some kind of an "acoustic-magnetospheric cyclotron accelerator". Using this conception we may provide a reasonable explanation for a number of satellite observations of occasional increases in inner zone particle fluxes (Gal'per et al., 1989; Gal'perin et al., 1992; Pustovetov and Malyshev, 1993, Bosková et al., 1994; Melioransky et al., 1996) which do not explicitly relate to solar or magnetospheric processes.

Summarizing we hope to draw the attention of space physicists to the possibility (practically never taken into account) that some of magnetospheric disturbances may be driven by processes in the upper atmosphere. Definitely, all the problems related to this problem cannot be solved in one paper, and further studies, both experimental and theoretical, are necessary.

Acknowledgements. This research was partially supported by the Commission of the European Union (grant no. INTAS-96-2064) and by the Russian Ministry of Science and Technology through the project "Space Research (Demeter)". One author (O.A.P.) wishes to thank the French Ministère de la Recherche et de la Technologie for hospitality at LPCE. We are greately appreciate the stimulating discussions with Dr. E. G. Stassinopoulos and Dr. J. I. Vette. Fruitful comments from the referee are acknowledged.

Topical Editor K.-H. Glassmeier thanks L. Stenflo and another referee for their help in evaluating this paper.

\section{References}

Al'perovich, L. S., B. O. Vugmeister, M. B. Gokhberg, V. L. Drobzev, V. I. Kazakov, and G. V. Fedorovich, About generation of geomagnetic variations by acoustical oscillations during earthquakes, Izv. Ac. of Sci. USSR (Physics of Solid Earth), 3, 58-68, 1979.

Al'perovich, L. S., E. A. Ponomarev, and G. V. Fedorovich, Geophysical phenomena modelled by an explosion: a review, Izv. Ac. of Sci. USSR (Physics of Solid Earth), 21, 816-825, 1985.

Belov, S. V., L. P. Gorbachev, and Yu. N. Savchenko, Generation of guided hydromagnetic signal by an acoustic wave pulse in an anisotropically conducting medium, Geomagn. Aeron., 13, 693698, 1973.

Blanc, E., Acoustical disturbances in the ionosphere: a review, Ann. Geophys., 3, 673-688, 1985.

Borisov, N. D., and B. S. Moiseyev, Generation of MHD disturbances in the ionosphere by a Rayleigh wave, Geomag. Aeron., 29, 472-476, 1989.

Bosková, J., J. Smilauer, P. Triska, and K. Kudela, Anomalous behavior of plasma parameters as observed by the Intercosmos 24 satellite prior to the Iranian earthquake of 20 June 1990 , Studia Geophys. Geod., 38, 213-220, 1994.

Cladis, J. B., Acceleration of geomagnetically trapped electrons by variations of ionospheric currents, J. Geophys. Res., 71, 5019$5025,1966$.
Danilov, A.V., and V. A. Dovzhenko, Excitation of electromagnetic fields by acoustic pulses entering the ionosphere, Geomag. Aeron., 27, 671-675, 1987.

Danilov, A. D., E. S. Kazimirovsky, G. V. Vergasova, and G. Yu. Khachikyan, Meteorological effects in the ionosphere, Gidrometeoizdat, Leningrad, 1987 (in Russian).

Falthammar, C. G., Effects of time-dependent electric fields on geomagnetically trapped radiation, J. Geophys. Res., 70, 24032516, 1965.

Fraser-Smith, A. C., Effects of man on geomagnetic activity and pulsations, Adv. Space Res., 1, 455-466, 1981.

Fukushima, N., Equivalence in ground magnetic effects of Chapman-Vestine's and Birkeland-Alfvén's electric current systems for polar magnetic storms, Rept. Ion. Space Res. Jap., 23, 219-227, 1969.

Gal'per, A. M., V. B. Dmitrienko, N. V. Nikitina, V. M. Grachev, and S. E. Ulin, Correlation between the high-energy charged particle fluxes in the radiation belt and seismicity of the Earth, Cosmic Res., 27, 789-792, 1989.

Gal'perin, Yu. I., and M. Hayakawa, On the magnetospheric effects of experimental ground explosions observed from AUREOL-3, J. Geomag. Geoelectr., 48, 1241-1263, 1996.

Gal'perin, Yu. I., V. A. Gladyshev, N. V. Dzhordzhio, R. A. Kovrazhkin, Yu. V. Lisakov, V. D. Maslov, L. M. Nikolayenko, R. Z. Sagdeev, O. A. Molchanov, M. M. Mogilevski, L. S. Al'perovich, M. B. Gokhberg, Ye. A. Ivanov, O. A. Pokhotelov, C. Beghin, J. J. Berthelier, J. M. Bosqued, and H. Reme, The Alfvén Wave excited in the middle-latitude magnetosphere by a large-scale acoustical wave which is propagated in the lower ionosphere, Izv. Ac. of Sci. USSR (Physics of Solid Earth), 22, 877-884, 1985.

Gal'perin, Yu. I., V. A. Gladyshev, N. V. Dzhordzhio, V. I. Larkina, and M. M. Mogilevski, Precipitation of high-energy captured particles in the magnetosphere above the epicenter of an incipient earthquake, Cosmic Res., 30, 89-106, 1992.

Goertz, C. K., and R. W. Boswell, Magnetosphere-ionosphere coupling, J. Geophys. Res., 84, 7239, 1979.

Gokhberg, M. B., V. A. Morgounov and O. A. Pokhotelov, Earthquake prediction: seismo-electromagnetic phenomena, Gordon and Breach, Reading, Philadelphia, 1995.

Guglielmi, A. V., and O. A. Pokhotelov, Geoelectromagnetic Waves, IOP Publishing, Bristol, Philadelphia, 1996.

Hayakawa, M., O. A. Molchanov, T. Ondoh, and E. Kawai, The precursory signature effect of the Kobe earthquake on VLF subionospheric signals, J. Comm. Res. Lab., 43, 169-180, 1996.

Imhof, W. L., and W. L. Smith, Observation of nearly monoenergetic high-energy electrons in the inner radiation belt, Phys. Rev. Lett., 14, 885-887, 1965.

Jacobson, A. R., A model for conjugate coupling from ionospheric dynamos in the acoustic frequency range, J. Geophys. Res., 91, 4404-4412, 1986.

Jacobson, A. R., and P. A. Bernhardt, Electrostatic effects in the coupling of upper atmospheric waves to ionospheric plasma, $J$. Geophys. Res., 90, 6533-6541, 1985.

Karlov, V. D., S. I. Kozlov, and V. P. Kudryavtsev, Large-scale disturbances in ionosphere emerging during the flight of a rocket with an operating engine, Cosmic Res., 18, 266-277, 1980.

Laaspere, T., W. C. Johnson, and L. C. Semprebon, Observations of auroral hiss, LHR noise, and other phenomena in the frequency range $20 \mathrm{~Hz}$ to $540 \mathrm{kHz}$ on OGO-6, J. Geophys. Res., 76, 44774493, 1971.

Larkina, V. I., A. V. Nalivayko, N. I. Gershenson, M. B. Gokhberg, V. A. Liperovskiy, and S. L. Shalimov, Observations of VLF emission, related with seismic activity, on the Interkosmos-19 satellite, Geomagn. Aeron., 23, 684-687, 1983.

Larkina, V. I., V. V. Migulin, O. A. Molchanov, V. B. Shvetsova, A. S. Inchin, I. A. Pimenov, and I. P. Khar'kov, Characteristics of the excitation of the low frequency emissions in the upper ionosphere above earthquake regions, Geomagn. Aeron., 28, 685-689, 1988. 
Lyons L. R., and D. J. Williams, Quantitave Aspects of Magnetospheric Physics, D. Reidel, Dordrecht, 1984.

Melioransky, A. S., T. N. Bibikova, and A. V. Belova, Hypothesis on influence of typhoons on the precipitation of electrons from Earth's radiation belts, Proceedings of the conference "Lithosphere-hydrosphere-atmosphere interaction", Moscow Univ., Moscow, 1996.

Molchanov, O. A., O. A. Mazhaeva, A. N. Goliavin, and M. Hayakawa, Observation by the Intercosmos-24 satellite of ELFVLF electromagnetic emissions associated with earthquakes, Ann. Geophys., 11, 431-440, 1993.

Molchanov, O. A., V. A. Rafalsky, and M. Hayakawa, Penetration characteristics of electromagnetic emissions from an underground seismic source into the atmosphere, ionosphere and magnetosphere, J. Geophys. Res., 100, 1691-1712, 1995.

Okuzawa, T., T. Shibata, and H. Yasui, On the ionospheric effect of near-source earthquakes around the islands of Japan detected by the HF-Doppler technique, J. Geomagn. Geoelectr., 35, 391$397,1983$.

Okuzawa, T., T. Shibata and T. Ichinose, Short-period disturbances in the ionosphere observed at the time of typhoons in September 1982 by a network of HF Doppler receivers, J. Geomagn. Geoelectr., 38, 239-266, 1986.
Parrot, M. and Y. Zaslavski, Physical mechanisms of man-made influences on the magnetosphere, Surv. Geophys., 17, 67-100, 1996.

Pokhotelov, O. A., V. A. Pilipenko, E. N. Fedorov, L. Stenflo, and P. K. Shukla, Induced electromagnetic turbulence in the ionosphere and the magnetosphere, Physica Scripta, 50, 600605, 1994.

Pokhotelov, O. A., M. Parrot, V. A. Pilipenko, E. N. Fedorov, V. V. Surkov, and V. A. Gladychev, Response of the ionosphere to natural and man-made acoustic sources, Ann. Geophys., 13, 10,197-10,210, 1995.

Popov, L. N., Yu. K. Krakovezkiy, M. B. Gokhberg, and V. A. Pilipenko, Terrogenic effects in the ionosphere: a review, Phys. Earth Planet. Inter., 57, 115-128, 1989.

Prasad, S. S., L. J. Schenk, and K. Davies, Ionospheric disturbances by severe tropospheric weather storms, J. Atmosph. Terr. Phys., 37, 1357-1363, 1975.

Pustovetov, V. P., and A. B. Malyshev, Space-time correlation of earthquakes and high-energy particle flux variations in the inner radiation belt, Cosmic Res., 31, 84-90, 1993.

Sorokin, V. M., and G. V. Fedorovich, Physics of slow MHD waves in the ionospheric plasma, Energoizdat, Moscow, 1982 (in Russian).

Sturrock P. A., Plasma Physics, Cambridge University Press, Cambridge, 1994. 